\title{
Navigating the current landscape of clinical genetic testing for inherited retinal dystrophies
}

\author{
Kristy Lee, $\mathrm{MS}^{1}$ and Seema Garg, MD, $\mathrm{PhD}^{2}$
}

Inherited eye disorders are a significant cause of vision loss. Genetic testing can be particularly helpful for patients with inherited retinal dystrophies because of genetic heterogeneity and overlapping phenotypes. The need to identify a molecular diagnosis for retinal dystrophies is particularly important in the era of developing novel gene therapy-based treatments, such as the RPE65 gene-based clinical trials and others on the horizon, as well as recent advances in reproductive options. The introduction of massively parallel sequencing technologies has significantly advanced the identification of novel gene candidates and has expanded the landscape of genetic testing. In a relatively short time clinical medicine has progressed from limited testing options to a plethora of choices ranging from single-gene testing to whole-exome sequencing. This article outlines currently available genetic testing and factors to consider when selecting appropriate testing for patients with inherited retinal dystrophies.

Genet Med advance online publication 19 March 2015

\section{INTRODUCTION}

Inherited eye diseases are a significant cause of vision loss. In the past few years, there has been an exponential increase in the identification of genetic mutations and loci for many ophthalmic conditions, including those described in the original research articles published in this issue. ${ }^{1,2}$ Some ophthalmic conditions are rare and monogenic, such as Best disease and transforming growth factor- $\beta 1$-related corneal dystrophies, and some are common with evidence of single-gene and multifactorial inheritance, such as age-related macular degeneration and late-onset primary open-angle glaucoma. At this time, the value of genetic testing for polygenic diseases has yet to be established and is relevant only in the research arena. When clinical findings suggest a known Mendelian disorder, however, the American Academy of Ophthalmology Task Force recommends genetic testing. ${ }^{3}$

Genetic testing for ophthalmic genetic disorders may provide an accurate, definitive diagnosis, helping to assess risk for the patient and family members, planning appropriate counseling, and determining inclusion in gene-based clinical trials.,4 Eden et al. ${ }^{5}$ interviewed 48 patients with retinitis pigmentosa (RP) and reported that $92 \%$ of participants desired genetic counseling and $86.5 \%$ desired genetic testing, demonstrating that most patients value these services. In ophthalmology, the first successful gene-based therapy was for Leber congenital amaurosis (LCA), an inherited retinal disorder causing infantile blindness. ${ }^{6,7}$ Because only those patients with a mutation in the RPE65 gene (OMIM 180069) would benefit from this therapy (17 genes are currently known to be associated with LCA), determining the molecular diagnosis was critical to determine inclusion in the trial. In addition to the LCA clinical trials, a growing number of gene-based therapies or prevention measures for inherited eye disorders are being studied. ${ }^{8}$

Inherited retinal dystrophies are the major cause of incurable familial blindness in the Western world and are characterized by degeneration of photoreceptor and retinal pigment epithelial cells. ${ }^{9}$ Over the past 25 years, more than 200 genes that cause inherited retinal dystrophies have been identified. ${ }^{10}$ Genetic testing is particularly important for patients with retinal dystrophies because genetic heterogeneity and overlapping phenotypes often preclude a specific diagnosis on clinical grounds alone. Few Mendelian disorders exhibit the degree of genetic heterogeneity demonstrated by RP, one of the most common retinal dystrophies, with a worldwide prevalence of 1 in $4,000 \cdot{ }^{11-13}$ Over 100 genes have been associated with this condition. ${ }^{14}$ Moreover, other retinal dystrophies, including cone-rod dystrophy (CRD), cone dystrophy, and Stargardt disease, also exhibit genetic heterogeneity. ${ }^{15,16}$ Inherited retinal dystrophies demonstrate significant phenotypic heterogeneity. For example, mutations in the ABCA4 gene (OMIM 601691) have been associated with several distinct hereditary retinal dystrophies and can have highly variable retinal appearance on clinical examination, ranging from normal to widespread pigmentary changes (Stargardt disease, CRD, cone dystrophy, and RP). ${ }^{12}$

Genetic testing options have greatly expanded rapidly over the past few years with the development of new technologies. Now multigene panels and whole-exome sequencing (exome sequencing) are available in addition to traditional Sanger sequencing and polymerase chain reaction-based testing. In the past genetic testing had a relatively low diagnostic yield in most patients with hereditary eye disease. For example, only $20-30 \%$ of patients with an autosomal-dominant form of RP

${ }^{1}$ Department of Genetics, University of North Carolina at Chapel Hill, Chapel Hill, North Carolina, USA; ${ }^{2}$ Department of Ophthalmology, University of North Carolina at Chapel Hill, Chapel Hill, North Carolina, USA. Correspondence: Kristy Lee (kristy_lee@med.unc.edu) 
(representing $15-25 \%$ of all patients with RP) had an identifiable molecular diagnosis based on single-gene testing for the RHO gene (OMIM 180380). ${ }^{12,17-19}$ As testing improved, small gene panels using Sanger sequencing became available in cases where both the specific clinical diagnosis (such as CRD) and the inheritance pattern was known. Given the complexities of inherited retinal disorders, however, these was not always known. With the advent of massively parallel sequencing (MPS) in the clinical setting, accurate molecular diagnosis is now possible for patients in whom the inheritance pattern and the clinical diagnosis are uncertain despite complete ophthalmologic evaluation, including biomicroscopy, perimetry, and electrophysiologic testing.

In the current landscape with a myriad of genetic testing options, how does a clinician decide which test to order? Factors to consider include the certainty of a patient's clinical diagnosis, the certainty of the pattern of inheritance, the turnaround time required, and the patient's insurance coverage. The goal of this article is to summarize the clinically available options and the factors to consider when determining appropriate genetic testing for single-gene retinal dystrophies.

\section{GENETIC TESTING OPTIONS}

With the inclusion of MPS technologies in CLIA-certified clinical laboratories, the options for genetic testing in patients with inherited retinal dystrophies are numerous. Currently available commonly ordered tests, with approximate turnaround times and costs, are listed in Table 1. The information provided in this table was obtained by surveying four to seven laboratories per test category (some tests are performed in fewer laboratories) and is intended to be a frame of reference for available testing at the time of this publication. Clinicians should consult the specific laboratory's website or personnel for accurate and up-to-date testing options, costs, and turnaround times when evaluating testing for their patients.

Although MPS technologies may provide a more comprehensive analysis, these tests may not always be the most appropriate or cost-effective if a more direct, targeted approach is available. A representative example is a patient with a relative who has a known molecular diagnosis; the most appropriate testing is for the known familial mutation(s). Confirmatory testing may become even more important in the future as human gene therapy trials and subsequent gene-based therapies become more commonplace. ${ }^{20,21}$ For rare and distinctive monogenic conditions, such as Best disease, which is known to be caused by only BEST1 (OMIM 607854), single-gene testing is most appropriate. Even in these apparent monogenic cases, however, more comprehensive testing could be considered if initial testing is negative. Chromosomal microarray to evaluate for copy-number variants or a contiguous gene deletion would be most appropriate for patients presenting with a retinal dystrophy in addition to other congenital anomalies, such as facial dysmorphism, developmental delay/intellectual disabilities, and/or other systemic diseases/malformations that do not seem to fit a known syndrome. ${ }^{12}$ A simultaneous karyotype analysis could also be considered; however, its diagnostic yield would be lower than that of a microarray analysis. ${ }^{22}$ Disease-specific Sanger or next-generation sequencing (NGS) gene panels are appropriate options for patients with a relatively certain clinical diagnosis that has genetic heterogeneity. These panels are available and include several common syndromic and nonsyndromic inherited retinal dystrophies, such as LCA, Usher syndrome, RP, CRD, and Stargardt disease. For a patient with a suspected inheritance pattern of nonsyndromic retinal dystrophies, smaller gene panels are available: autosomal-dominant, autosomal-recessive, and $\mathrm{X}$-linked gene panels. In simplex cases (i.e., cases with a negative

Table 1 Clinically available molecular testing for retinal disorders

\begin{tabular}{|c|c|c|c|}
\hline Type of testing & Indication & $\begin{array}{l}\text { Turnaround } \\
\text { time (weeks) }\end{array}$ & Approximate cost ${ }^{a}$ \\
\hline Sanger sequencing & - Clinical diagnosis of a monogenic disorder & $3-6$ & $\$ 200-2,000^{\mathrm{b}}$ \\
\hline Mitochondrial genome sequencing & $\begin{array}{l}\text { - Clinical suspicion of an unknown mitochondrial } \\
\text { syndrome }\end{array}$ & $7-8$ & $\sim \$ 3,000$ \\
\hline $\begin{array}{l}\text { Disease-specific Sanger sequencing or } \\
\text { NGS gene panels }\end{array}$ & - Clinical diagnosis of a heterogeneous disorder & $6-12$ & $\$ 300-4,000^{c}$ \\
\hline Array CGH deletion/duplication panels & $\begin{array}{l}\text { - Clinical diagnosis of a heterogeneous disorder } \\
\text { - Ordered in conjunction with or after negative (or } \\
\text { only one AR variant) NGS panels }\end{array}$ & $2-4$ & $\$ 650-1,300$ \\
\hline Comprehensive NGS retina gene panels & $\begin{array}{l}\text { - Clinical diagnosis of a heterogeneous disorder } \\
\text { - Uncertain diagnosis }\end{array}$ & 12 & $\sim \$ 2,500$ \\
\hline Whole-exome sequencing & $\begin{array}{l}\text { - Clinical diagnosis of a heterogeneous disorder } \\
\text { - Uncertain diagnosis } \\
\text { - Negative findings on previous NGS }\end{array}$ & $12-24$ & $\$ 5,000-7,000^{d}$ \\
\hline
\end{tabular}

AR, autosomal recessive; CGH, comparative genomic hybridization; NGS, next-generation sequencing; SNP, single-nucleotide polymorphism.

aThe "approximate cost" was derived by surveying four to seven laboratories offering each specific test to provide a frame of reference. Clinicians should consult laboratory personnel or websites for up-to-date pricing. bPrice variation largely reflects the number of exons required to sequence. 'Price variation largely reflects the number of genes

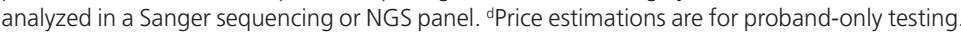


family history), however, it is difficult to distinguish between recessive inheritance and dominant disorders with incomplete penetrance or a de novo mutation; thus, a broader disease gene panel may be preferable to an autosomal-recessive gene panel. For example, $\mathrm{RHO}$ exhibits a higher rate of de novo mutations than previously appreciated, and PRPH2 (OMIM 179605) is associated with incomplete penetrance. $12,17,19,23,24$

For patients without a definitive clinical diagnosis or patients suspected of having a condition with high locus heterogeneity without a clear pattern of inheritance, comprehensive NGS panels, often referred to as "retinal dystrophy panels," are commercially available. These panels typically include sequencing of more than 100 genes known to be associated with syndromic and nonsyndromic retinal dystrophies.

The vast majority of pathogenic variants in retinal dystrophies are not likely to be large gene deletions. However, some genes, such as PRPF31 (OMIM 60419), which is associated with autosomal-dominant RP, do have a higher percentage of deletions that would be missed on sequencing panels. ${ }^{25}$ Because Sanger sequencing is unable and NGS platforms are not currently optimized to detect copy-number changes, commercial laboratories also offer disease-specific deletion and duplication panels to complement the sequencing panels. ${ }^{12}$ Several commercial laboratories offer a gene-targeted comparative genomic hybridization array to identify larger deletions and duplications in all of the genes included in their sequencing panels. Most commercial laboratories also still offer single-gene dosage assays to detect possible large/whole-gene deletions. Therefore, when turnaround time for results is not a factor, one could order a single-gene deletion/duplication analysis for patients who have only one identifiable variant in a gene associated with an autosomal-recessive condition, as opposed to ordering simultaneously a comparative genomic hybridization analysis and a Sanger or NGS gene panel.

The most comprehensive testing performed by commercial laboratories is exome sequencing. This testing could be considered for a patient without a clear clinical diagnosis; a patient with a condition with high locus heterogeneity, such as RP; or a patient who previously had negative genetic testing or whose affected relative(s) previously had negative comprehensive testing. Testing options include proband-only or trio (patient plus parents) testing in the setting of a simplex case, or testing of other affected relatives, if available. Trio testing can increase the detection rate of exome sequencing by allowing the laboratory to set phase for variants associated with autosomal-recessive conditions and to investigate the possibility of a de novo variant when identified in a gene associated with an autosomal-dominant condition and no known family history. ${ }^{26}$ However, trio testing is not always possible in families from which relatives may be unavailable, such as in the case of adoption, or are unwilling to undergo testing. Proband-only testing can still be informative, but it may lead to a higher percentage of variants of unknown significance. In addition, recognizing that panel testing is still likely to be superior to exome sequencing in terms of analytic sensitivity and specificity, and that the added clinical usefulness of exome sequencing after known disease genes have been analyzed by a large panel is lower, is important..$^{20}$ The cost of exome sequencing can vary between trio and proband-only testing. Some laboratories charge the same price for trio and proband-only testing and have a higher base rate, whereas other laboratories have a lower base rate and charge up to $\$ 2,500$ per relative.

For patients in whom a specific mitochondrial condition is suspected because of other systemic disease, testing for individual mitochondrial conditions is available. For patients in whom a mitochondrial disorder is suspected but the phenotype is not suggestive of a particular syndrome, sequencing of the mitochondrial genome may be considered. There are few known cases of mitochondrial nonsyndromic retinopathies. ${ }^{27}$ Thus, this testing may be more appropriate in the research setting for patients with previously negative comprehensive testing.

Decisions regarding genetic testing must be considered on an individual patient basis. Figure 1 demonstrates a proposed a genetic testing schema for common patient scenarios encountered in a typical genetics or ophthalmic genetics clinic.

\section{DIAGNOSTIC YIELD OF MPS TESTING}

The gene discoveries facilitated by MPS technologies in the research setting have certainly improved the diagnostic yield of genetic testing in the clinical setting. ${ }^{23}$ Several researchers have investigated the diagnostic yield of MPS technologies in patients with a variety of inherited retinal dystrophies. Using exome sequencing, $\mathrm{Xu}$ et al. ${ }^{28}$ found known or likely deleterious variants in 79 of 157 (50\%) of families with RP, representing autosomal-dominant, autosomal-recessive, and X-linked patterns of inheritance. Similarly, a detection rate of $\sim 50-70 \%$ with MPS technologies was noted in several previous studies., ${ }^{1,17,23}$ Most recently, Corton et al., ${ }^{29}$ using exome sequencing, found causative variants in 10 of 12 families (83\%), with a variety of clinical phenotypes, including RP, Usher syndrome, choroideremia, and Stargardt disease. Using NGS gene panels containing 163 genes previously known to be associated with syndromic and nonsyndromic retinopathies, Wang et al. ${ }^{30}$ found a causative mutation in $37 \%$ of 123 patients with RP. Using a larger NGS panel of 254 known and candidate genes in 14 patients with a variety of retinopathies, including RP, Stargardt disease, and congenital stationary night blindness, Audo et al. ${ }^{31}$ reported a 57\% detection rate. Although using limited sample sizes, these studies demonstrate the effectiveness of MPS techniques to determine the molecular etiology of a variety of retinal phenotypes. A caveats to consider with respect to these studies is that the reported diagnostic yield is heavily influenced by the selection of patients being tested (the prior probability of finding a Mendelian disorder), by the proportion of cases that are attributable to known disease genes (clinical sensitivity of testing for that condition), and by the interpretation of variants (in some cases pathogenicity was suggested without cosegregation data or other supporting evidence). While these studies provide a baseline for comparison, the reported yields may be inflated by ascertainment bias and thus may not translate directly to testing performed in a routine clinical setting. The significant percentage of patients with negative MPS analyses suggest that there 


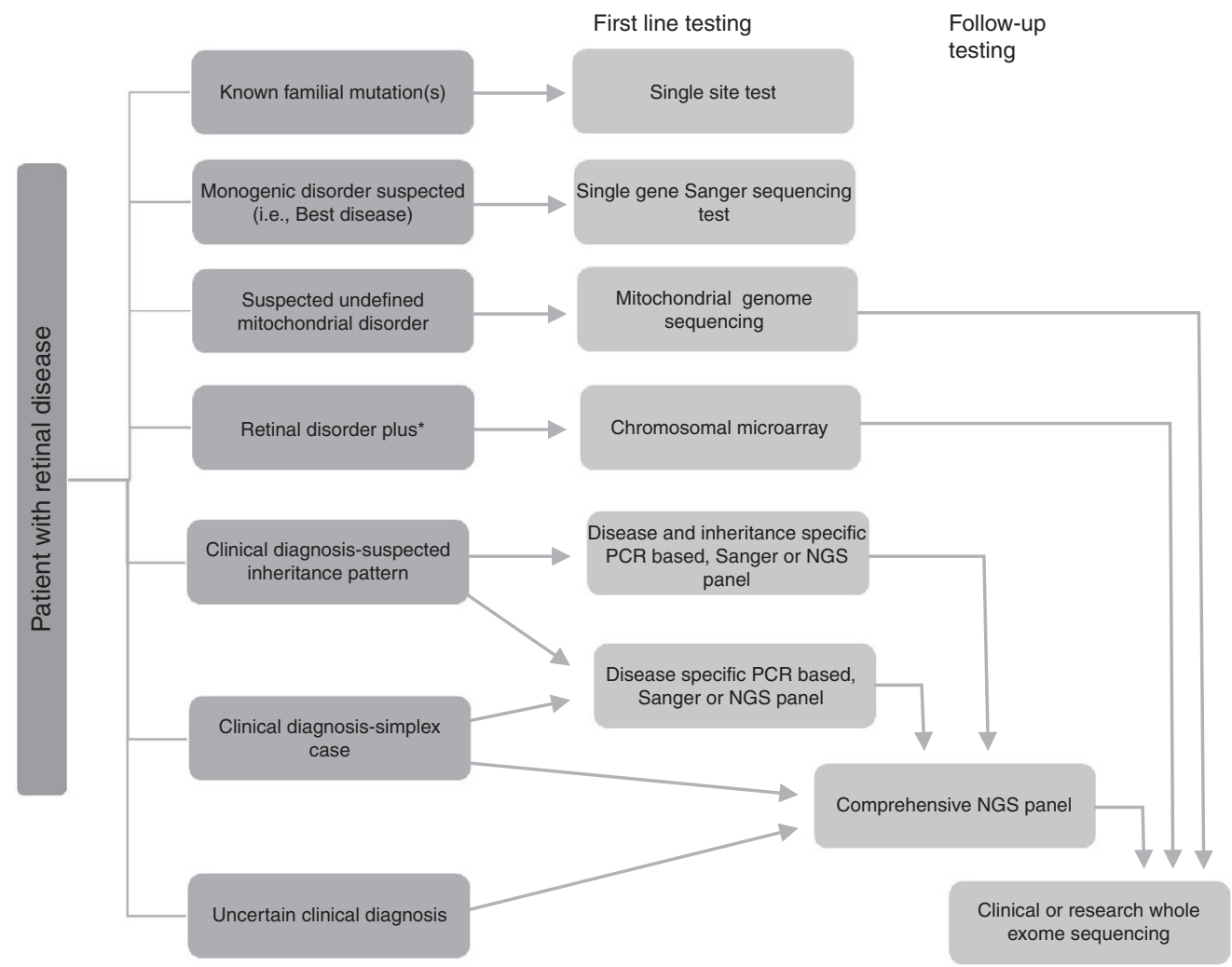

Figure 1 Genetic testing options for patients with retinal dystrophies. Genetic testing for patients with retinal disorders should be considered on a case-by-case basis. This schema illustrates available options for initial genetic testing and follow-up analysis, if required. *Retinal disorder plus refers to patient with a retinal dystrophy in combination with additional systemic features, developmental delay/intellectual disabilities, and/or congenital malformations. NGS, next-generation sequencing; PCR, polymerase chain reaction.

are additional as-yet undiscovered genes associated with retinal dystrophies or more complex genetic etiologies that have not been fully explored. ${ }^{12}$ That the reported detection rates seem to be similar between studies using exome sequencing and those using NGS gene panels is also worth noting. This suggests that, at this time, exome sequencing may not add a tremendous diagnostic value over NGS gene panels in the clinical setting.

\section{Expanding clinical phenotypes}

\section{ADVANTAGES OF MPS TESTING}

The use of MPS techniques in the research setting has uncovered previously unrecognized clinical phenotypes associated with individual genes. For example, null mutations in the ABHD12 gene (OMIM 613599) have been previously associated with PHARC syndrome (polyneuropathy, hearing loss, cerebellar ataxia, RP, and cataracts). ${ }^{32}$ Exome sequencing of individuals with a clinical diagnosis of nonsyndromic, autosomal-recessive RP (arRP) identified five novel variants in the $A B H D 12$ gene that cosegregated with disease, which expand the phenotype associated with this gene to include nonsyndromic RP. ${ }^{33}$ In another example, the RPE65 gene was associated with autosomal-recessive LCA and arRP. ${ }^{34,35}$ With the use of linkage analysis and exome sequencing, a dominant-negative mutation was detected in a large family with autosomaldominant RP. ${ }^{36}$ The RPE65 variant cosegregated with disease in all 20 affected family members within this four-generation family, thus expanding the previously described recessive phenotype of RPE65 retinal dystrophies. Bowne et al. ${ }^{36}$ reported the same RPE65 variant in a father and two daughters with a clinical diagnosis of choroideremia and no identifiable deleterious variants in the $C H M$ gene, the only gene previously known to be associated with choroideremia. They suggested that RPE65 variants also be considered in patients with retinal degeneration and choroidal involvement. RPE65 is not the only gene reported to contain variants causing both dominant and recessive patterns of inheritance. Loss-of-function variants associated with recessive disease and dominant-negative variants, gain-of-function variants, or variants leading to haploinsufficiency have been associated with autosomal-dominant patterns of inheritance in BEST1, NR2E3 (OMIM 604485), NRL (OMIM 162080), RP1 (OMIM 603937) and RHO genes. ${ }^{23,37,38}$

Truncating variants within the EYS gene (OMIM 612424) were initially associated with arRP in $2008 .^{39,40}$ Novel truncating variants were identified in a patient with a clinical diagnosis of autosomal-recessive CRD. ${ }^{41}$ Parental testing confirmed that these variants were in trans. This finding expands the known phenotype associated with the EYS gene to include autosomalrecessive CRD.

As more patients are sequenced with MPS technology, associated clinical phenotypes will continue to expand. Many 
conditions with overlapping features, such as CRD and cone dystrophy, likely represent a mutational spectrum within a particular gene. Cataloging variants in these genes is important to establish phenotype/genotype correlations. ${ }^{42}$ In addition, certain types of variants (such as dominant-negative variants) could represent an autosomal-dominant form of a disease that is known to be inherited in an autosomal-recessive fashion.

\section{Atypical, early, and evolving presentations}

MPS testing has provided insight into the conditions of many patients with a previous diagnosis of "retinal dystrophy not otherwise specified" or "atypical retinitis pigmentosa" and in whom limited panel testing would be less effective. For example, exome sequencing identified compound heterozygous variants in the C8orf37 gene (OMIM 614477) in two siblings with severe, earlyonset retinal dystrophy with macular atrophy, cataracts, and high myopia. ${ }^{43}$ Deleterious variants in the C8orf37 gene have previously been associated with early-onset arRP and autosomalrecessive CRD ${ }^{44,45}$ Katagiri et al. ${ }^{43}$ reported that the progression and severity of the siblings' retinopathy precluded them from distinguishing between RP and CRD as the children's phenotype. Broad retinal dystrophy panel testing reduces the importance of a definitive clinical diagnosis to determine the appropriate test.

Phenotypic variability of inherited retinal dystrophies within the same family is common and can present diagnostic dilemmas. Goldenberg-Cohen et al. ${ }^{46}$ reported a consanguineous family with two siblings exhibiting an Usher syndrome phenotype, one with isolated RP and one who was unaffected. Exome sequencing identified two separate retinal disorders segregating in this family: a homozygous variant in the MYO7A gene (OMIM 276903) associated with Usher syndrome in the two siblings with an Usher syndrome phenotype and a homozygous frame-shifting variant in the PDE6B gene (OMIM 180072) in the sibling with isolated RP. One of the two siblings with Usher syndrome was also homozygous for the PDE6B variant, which would be critical information when providing genetic counseling to this individual and any of their future children because one might have assumed that the RP features would be consistent with the Usher syndrome phenotype. The unaffected sibling was a carrier for the $P D E 6 B$ variant and homozygous wild-type for MYO7A, and the parents were both carriers of each variant. While having a family with two distinct retinal dystrophies is uncommon, even in consanguineous families, this example illustrates that broad MPS testing may be helpful in families with wide phenotypic variability.

Evolving phenotypes can complicate making an accurate clinical diagnosis. For example, in nonsyndromic cases, some patients initially diagnosed with Stargardt disease later develop degeneration of rod photoreceptors and are subsequently diagnosed with a different retinopathy, such as CRD. ${ }^{4}$ The retinal manifestations in some syndromic cases may not be present early on, and an early diagnosis before symptoms begin may prove to be vital as preventative treatments become available. Prokudin et al..$^{47}$ presented an example of two siblings with an apparent syndromic condition, in whom the clinical diagnosis became clearer as the children developed more characteristic features of the condition. During their initial evaluation at ages 4 and 1 years, microcephaly and developmental delay were noted during the clinical exam, and an appropriate genetic workup yielded no diagnosis at that time. Three years later, exome sequencing was performed on both siblings and their parents, and a homozygous variant was detected in the VPS13B gene (OMIM 607817), consistent with Cohen syndrome. Repeat clinical examination of both children at the time of results showed additional features suggestive of Cohen syndrome, including short stature, brachydactyly, conductive hearing loss, facial dysmorphia, pigmentary retinopathy, and bull's eye maculopathy. This case illustrates that phenotypes can evolve over time and that exome sequencing can enable a diagnosis without the limitation of presenting clinical features at the time of testing, which may decrease the time to diagnosis.

In clinical practice it is not uncommon to have a patient that has abnormal but nonspecific features of a retinal dystrophy or enough symptoms to meet the diagnostic criteria for a specific genetic condition. While a correct diagnosis may not impact treatment options at that time, the lack of a diagnosis can be stressful for patients and precludes appropriate genetic counseling and accurate risk assessment. ${ }^{2,5} \mathrm{~A}$ broad molecular DNA test along with results from ancillary ophthalmologic testing may greatly enhance a clinician's ability to provide an accurate diagnosis and thus guide management.

\section{SPECIAL CONSIDERATIONS WITH MPS TESTING} Variant interpretation

Difficulties in variant interpretation is not a new issue unique to MPS technologies; however, the scale of possible variants is massive. As more sequencing data become public through the efforts of data sharing initiatives, knowledge of these variants will continue to increase over time..$^{23,48}$ Laboratories performing clinical MPS testing should ensure that proper evidence is in place to classify the variant as pathogenic, such as cosegregation data, the lack of a variant in healthy family members in published reports, the absence or small representation of the variant in large control data sets (e.g., dbSNP, Exome Variant Server, and ExAc Browser), and in vitro data that show aberrant gene function. ${ }^{42,49-55}$ Many variants that have previously been reported as pathogenic in the medical literature (and thus are represented in databases used for molecular DNA analysis) are now known to have allele frequencies that make them very common occurrences in the general population, or they have been seen in the homozygous state in healthy controls. ${ }^{56-58}$ Large polymorphic genes, such as the $A B C A 4$ gene associated with Stargardt disease, arRP, autosomal-recessive CRD, and cone dystrophy, are likely to contain more variants that require scrutiny because some variants may have been previously erroneously asserted as being pathogenic. ${ }^{59}$

When the appropriate level of evidence is not present to classify a variant as known/likely benign or deleterious, the laboratory must classify the variant as a "variant of unknown significance." The clinician must provide adequate counseling 
before testing to educate patients that larger gene panels may be associated with a higher number of variants of unknown significance that could be difficult to interpret because gene variants are common, and larger panels may include genes for which less clinical data are available or known.

Clinicians also need to carefully review laboratory reports asserting pathogenic, likely pathogenic, or variants of unknown significance findings to ensure that the variant(s) implicated is associated with the patient's phenotype. With broad panels there is the possibility that the patient could be a carrier for a different form of retinal dystrophy. Thus having one pathogenic mutation in a condition associated with an autosomal-recessive inheritance could mean the patient is a carrier for that condition or that a mutation in the other allele was not identified. There is also a possibility that a patient could have one or more suspicious variants that does not fit the phenotype. Because laboratorians often have limited information regarding the patient's phenotype, clinical correlation by the clinician is essential. ${ }^{60}$ The complex nature of genetic results from large panels or genomescale sequencing may necessitate a multidisciplinary approach that incorporates the input of specialists in clinical genetics.

\section{Limitations of MPS testing}

The detection of certain types of mutations, such as trinucleotide repeats, small insertions/deletions, and copy-number variants, can be missed by MPS technology because of homopolymer runs, the presence of pseudo-genes, or the repetitive nature of the specific region of a gene. ${ }^{49,61}$ By searching for variants in known positive controls, Audo et al. ${ }^{31}$ studied the robustness of NGS panels and found lower coverage for two genes associated with congenital stationary night blindness, NYX (OMIM 300278) and GRM6 (OMIM 604096), as a result of GC-rich regions. In addition, because of low coverage of the PRPF31 gene, a known one-base pair deletion was undetectable. However, Consugar et al..$^{20}$ demonstrated that, overall, a selective targeted enrichment with NGS panels outperforms exome sequencing. The improved detection rate was attributed to probe design that could be tailored to genes associated with retinal dystrophies; probes for approximately $10 \%$ of the regions targeted by their NGS panel were missing from commercially available exome capture kits. They also suggested that the turnaround time is greatly improved by using the targeted panel versus exome sequencing.

Another limitation of MPS technologies is detection of deleterious variants in the RPGR gene (OMIM 312610) because of its repetitive region of open reading frame $15 .{ }^{31}$ Using exome sequencing, however, Bassuk et al. ${ }^{62}$ reported an apparent deleterious variant in the open reading frame 15 region of the RPGR gene in a patient with a suspected diagnosis of Stargardt disease. The variant was subsequently confirmed via Sanger sequencing. Therefore, in cases in which X-linked inheritance is suggested or in a male with simplex RP, CRD, or cone dystrophy, a clinician may consider the option of Sanger sequencing for the RPGR gene.

While the detection of deleterious variants within genes that are more difficult to sequence may be possible, noting that these genes have a decreased yield when analyzed with MPS technologies is important. In the event of a negative sequencing analysis, the exon coverage within genes potentially associated with the patient's phenotype should be specifically examined. Any gene(s) of particular interest to the clinician may require additional testing by Sanger sequencing if there is concern about a false-negative result. Furthermore, analysis to determine duplications or deletions may be necessary in patients with only one identifiable deleterious variant associated with a recessively inherited condition of interest.

\section{Incidental or secondary findings}

The possibility of incidental or secondary findings unrelated to the primary indication for sequencing (e.g., retinal dystrophy) adds another major layer of complexity to exome sequencing over traditional genetic testing and NGS gene panels. ${ }^{2}$ In the clinical setting incidental findings can be categorized in different ways, often relating to the clinical actionability of such findings. ${ }^{63}$ Medically actionable incidental findings refer to conditions that have a preventative intervention or treatment as opposed to non-medically actionable findings that may provide information but have no intervention at this time. In 2013 the American College of Medical Genetics and Genomics published a recommendation that laboratories return medically actionable incidental findings when a clinical exome or genome analysis is ordered ${ }^{64}$ Most CLIA-certified clinical laboratories also offer patients the option of learning non-medically actionable findings. Therefore, exome sequencing requires a thorough and detailed discussion regarding what results, if any, a patient would like to receive..$^{60}$ Laboratories require patients to indicate their preferences for learning different types of results on the testing consent form, and clinicians must document that these options have been reviewed with the patient. ${ }^{60}$ In addition to the extra time needed for the informed consent process, exome sequencing requires the clinician to spend additional time interpreting laboratory reports, preparing for results, and reviewing results with patients because patients will likely have results that are less familiar to the clinician. ${ }^{2,60}$ The application of exome sequencing mandates a clinical provider who is capable of managing this complex test, and clinicians lacking appropriate training and expertise should consider a referral to a genetics specialist with more experience ordering exome sequencing or potentially refer patients to a research study offering exome sequencing.

\section{COST CONSIDERATIONS}

Costs of genetic testing and insurance coverage are important factors in the choice of genetic testing options. The cost of comprehensive NSG gene panels is quickly becoming equivalent to that of smaller NGS panels, and the cost of clinical exome sequencing is also decreasing. Several insurance companies reimburse the costs of genetic testing, which affords many patients the option of genetic testing. However, some insurance carriers, such as Cigna Corporation, require that genetic testing be ordered by a board-certified medical geneticist or a board-certified genetic counselor to be a covered 
benefit. In addition, more traditional types of testing, such as single-gene analysis and gene panel testing, are more likely to be covered than exome sequencing; some insurance companies still consider this newer technology to be a research-grade test. ${ }^{54}$ Several testing labs offer pre-verification services as well as reduced cost and payment plans for qualifying patients. Patients should be aware of the potential cost, and clinicians may consider providing patients with diagnostic codes and Current Procedural Terminology billing codes to allow them the opportunity to contact their insurance company for coverage and copayment information before testing. With the proper documentation by the ordering provider describing the potential medical benefit of the test (often a letter of medical necessity or a form provided by the insurance company), an insurance carrier may also offer the patient and laboratory a verification of benefits before genetic testing. A UK study found that over half of participants with a diagnosis of RP were willing to pay over $£ 500$ (approximately US\$780) for genetic counseling and genetic testing services. ${ }^{5}$ Some patients place a high value on these services and may be willing to pay out-ofpocket fees to obtain them, if necessary.

Research testing may represent another option for some patients. The National Eye Institute's eyeGENE Program, started in 2006, provides genetic testing for several inherited eye disorders, including retinal dystrophies, in a research setting, with confirmation of known or likely disease-causing variants by a CLIA-approved laboratory at no cost to the patient. The program requires the clinician to provide detailed phenotype information to better characterize the natural history and establish genotypephenotype correlations (additional information can be found at https://www.nei.nih.gov/eyegene). In addition, the National Human Genetic Research Institute's Centers for Mendelian Genomics accepts referrals from health-care providers who have patients with a suspected Mendelian disorder of unknown etiology (for additional information go to http://mendelian.org). ${ }^{65}$ Other important research efforts to better characterize the natural history and/or the molecular etiology of a variety of inherited retinal diseases are ongoing at several academic institutions (for additional information go to http://www.clinicaltrials.gov).

\section{SUMMARY}

In the span of a relatively short time, clinical medicine has progressed from few genetic testing options for patients with inherited retinal dystrophy to a plethora of choices ranging from single-gene testing to whole-exome sequencing. In addition to choosing the appropriate testing for a given patient, clinicians must also take into consideration his or her ability to appropriately counsel the patient both before and after testing, as well as to accurately interpret the test results and correlate findings with the patient's unique phenotype. While MPS technologies are not necessarily superior to more traditional testing, they do offer distinct advantages in some clinical scenarios, such as for patients with highly heterogeneous disorders, such as RP and $\mathrm{CRD}$, and patients with an unclear clinical diagnosis. Having a thorough understanding of the strengths and limitations of the various testing methodologies for different retinal disorders is key to choosing appropriate testing.

Deciding the most appropriate genetic test methodology to use to evaluate a patient can be difficult given the plethora of expanded options within recent years. While one could argueand some experts do-that exome sequencing should be used primarily for gene discovery in a research context and not as a first-line clinical test, even choosing between gene panels is not always straightforward. ${ }^{20,54,60}$ The clinician's goal is to order the fewest tests with the highest likelihood of identifying the underlying etiology. Broad-scope testing for more genes may not be cost-effective and could reveal unrelated, secondary information. Future directions for research should involve examining the cost-effectiveness of different MPS testing strategies versus traditional polymerase chain reaction-based and Sanger sequencing panels and patient preferences for genetic testing, which could then guide clinicians regarding the most appropriate genetic test for the complex arena of inherited retinal diseases.

\section{ACKNOWLEDGMENTS}

The authors thank Julianne O'Daniel, MS, CGC, and Jonathan Berg, MD, PhD, for their thoughtful review of this manuscript. The authors also acknowledge Research to Prevent Blindness, New York, NY, and the Bryson Program for Human Genetics at the University of North Carolina at Chapel Hill for their continued support.

\section{DISCLOSURE}

The authors declare no conflict of interest.

\section{REFERENCES}

1. O'Sullivan J, Mullaney BG, Bhaskar SS, et al. A paradigm shift in the delivery of services for diagnosis of inherited retinal disease. J Med Genet 2012;49:322-326.

2. Gillespie RL, Hall G, Black GC. Genetic testing for inherited ocular disease: delivering on the promise at last? Clin Experiment Ophthalmol 2014;42:65-77.

3. Recommendations of the American Academy of Ophthalmology Task Force on Genetic Testing, 2014. http://one.aao.org/clinical-statement/recommendationsgenetic-testing-of-inherited-eye-d. Accessed 18 November 2014.

4. Zhang X, Ge X, Shi W, et al. Molecular diagnosis of putative Stargardt disease by capture next generation sequencing. PLOS ONE 2014;9:e95528.

5. Eden M, Payne K, Combs RM, Hall G, McAllister M, Black GC. Valuing the benefits of genetic testing for retinitis pigmentosa: a pilot application of the contingent valuation method. Br J Ophthalmol 2013;97:1051-1056.

6. Maguire AM, Simonelli F, Pierce EA, et al. Safety and efficacy of gene transfer for Leber's congenital amaurosis. N Engl J Med 2008;358:2240-2248.

7. Testa F, Maguire AM, Rossi S, et al. Three-year follow-up after unilateral subretinal delivery of adeno-associated virus in patients with Leber congenital Amaurosis type 2. Ophthalmology 2013;120:1283-1291.

8. ClinicalTrials.gov. http://clinicaltrials.gov. Accessed 19 November, 2014.

9. den Hollander Al, Black A, Bennett J, Cremers FP. Lighting a candle in the dark: advances in genetics and gene therapy of recessive retinal dystrophies. J Clin Invest 2010;120:3042-3053

10. RetNet. http://www.sph.uth.tmc.edu/RetNet. Accessed 21 November 2014.

11. Chang S, Vaccarella L, Olatunji S, Cebulla C, Christoforidis J. Diagnostic challenges in retinitis pigmentosa: genotypic multiplicity and phenotypic variability. Curr Genomics 2011;12:267-275.

12. Anasagasti A, Irigoyen C, Barandika O, López de Munain A, Ruiz-Ederra J. Current mutation discovery approaches in Retinitis Pigmentosa. Vision Res 2012;75:117-129.

13. Hartong DT, Berson EL, Dryja TP. Retinitis pigmentosa. Lancet 2006;368: 1795-1809 
14. Chizzolini M, Galan A, Milan E, Sebastiani A, Costagliola C, Parmeggiani F. Good epidemiologic practice in retinitis pigmentosa: from phenotyping to biobanking. Curr Genomics 2011;12:260-266.

15. Berger W, Kloeckener-Gruissem B, Neidhardt J. The molecular basis of human retinal and vitreoretinal diseases. Prog Retin Eye Res 2010;29:335-375.

16. Michaelides M, Hardcastle AJ, Hunt DM, Moore AT. Progressive cone and conerod dystrophies: phenotypes and underlying molecular genetic basis. Surv Ophthalmol 2006;51:232-258.

17. Neveling K, Collin RW, Gilissen C, et al. Next-generation genetic testing for retinitis pigmentosa. Hum Mutat 2012;33:963-972.

18. Daiger SP, Bowne SJ, Sullivan LS. Perspective on genes and mutations causing retinitis pigmentosa. Arch Ophthalmol 2007;125:151-158.

19. Daiger SP, Sullivan LS, Gire AI, Birch DG, Heckenlively JR, Bowne SJ. Mutations in known genes account for $58 \%$ of autosomal dominant retinitis pigmentosa (adRP). Adv Exp Med Biol 2008;613:203-209.

20. Consugar MB, Navarro-Gomez D, Place EM, et al. Panel-based genetic diagnostic testing for inherited eye diseases is highly accurate and reproducible, and more sensitive for variant detection, than exome sequencing. Genet Med 2015;17:253-261.

21. Wiggs JL, Pierce EA. Genetic testing for inherited eye disease: who benefits? JAMA Ophthalmol 2013;131:1265-1266.

22. Miller DT, Adam MP, Aradhya S, et al. Consensus statement: chromosomal microarray is a first-tier clinical diagnostic test for individuals with developmental disabilities or congenital anomalies. Am J Hum Genet 2010;86:749-764.

23. Davies WIL. Challenges using diagnostic next-generation sequencing in the clinical environment for inherited retinal disorders. Per Med 2014;11:99-111.

24. Krämer F, White K, Pauleikhoff $D$, et al. Mutations in the VMD2 gene are associated with juvenile-onset vitelliform macular dystrophy (Best disease) and adult vitelliform macular dystrophy but not age-related macular degeneration. Eur J Hum Genet 2000;8:286-292.

25. Sullivan LS, Bowne SJ, Seaman CR, et al. Genomic rearrangements of the PRPF31 gene account for $2.5 \%$ of autosomal dominant retinitis pigmentosa. Invest Ophthalmol Vis Sci 2006;47:4579-4588.

26. Lee $H$, Deignan JL, Dorrani $N$, et al. Clinical exome sequencing for genetic identification of rare Mendelian disorders. JAMA 2014;312:1880-1887.

27. Retinitis Pigmentosa Overview, 2014. http://www.ncbi.nlm.nih.gov/books/ NBK1417. Accessed 18 November 2014.

28. Xu Y, Guan L, Shen T, et al. Mutations of 60 known causative genes in 157 families with retinitis pigmentosa based on exome sequencing. Hum Genet 2014;133:1255-1271.

29. Corton M, Nishiguchi KM, Avila-Fernández A, et al. Exome sequencing of index patients with retinal dystrophies as a tool for molecular diagnosis. PLOS ONE 2013:8:e65574.

30. Wang $\mathrm{F}$, Wang $\mathrm{H}$, Tuan $\mathrm{HF}$, et al. Next generation sequencing-based molecular diagnosis of retinitis pigmentosa: identification of a novel genotype-phenotype correlation and clinical refinements. Hum Genet 2014;133:331-345.

31. Audo I, Bujakowska KM, Léveillard T, et al. Development and application of a next-generation-sequencing (NGS) approach to detect known and novel gene defects underlying retinal diseases. Orphanet J Rare Dis 2012;7:8.

32. Fiskerstrand $T, H^{\prime}$ mida-Ben Brahim D, Johansson S, et al. Mutations in ABHD12 cause the neurodegenerative disease PHARC: An inborn error of endocannabinoid metabolism. Am J Hum Genet 2010;87:410-417.

33. Nishiguchi KM, Avila-Fernandez A, van Huet RA, et al. Exome sequencing extends the phenotypic spectrum for ABHD12 mutations: from syndromic to nonsyndromic retinal degeneration. Ophthalmology 2014;121:1620-1627.

34. Marlhens F, Bareil C, Griffoin JM, et al. Mutations in RPE65 cause Leber's congenital amaurosis. Nat Genet 1997;17:139-141.

35. Morimura H, Fishman GA, Grover SA, Fulton AB, Berson EL, Dryja TP. Mutations in the RPE65 gene in patients with autosomal recessive retinitis pigmentosa or leber congenital amaurosis. Proc Natl Acad Sci USA 1998;95:3088-3093.

36. Bowne SJ, Humphries MM, Sullivan LS, et al. A dominant mutation in RPE65 identified by whole-exome sequencing causes retinitis pigmentosa with choroidal involvement. Eur J Hum Genet 2011;19:1074-1081.

37. Daiger SP, Sullivan LS, Bowne SJ. Genes and mutations causing retinitis pigmentosa. Clin Genet 2013;84:132-141.

38. Rattner A, Sun $\mathrm{H}$, Nathans J. Molecular genetics of human retinal disease. Annu Rev Genet 1999;33:89-131.

39. Abd El-Aziz MM, Barragan I, O'Driscoll CA, et al. EYS, encoding an ortholog of Drosophila spacemaker, is mutated in autosomal recessive retinitis pigmentosa. Nat Genet 2008;40:1285-1287.

40. Collin RW, Littink KW, Klevering BJ, et al. Identification of a $2 \mathrm{Mb}$ human ortholog of Drosophila eyes shut/spacemaker that is mutated in patients with retinitis pigmentosa. Am J Hum Genet 2008;83:594-603.
41. Katagiri S, Akahori M, Hayashi T, et al. Autosomal recessive cone-rod dystrophy associated with compound heterozygous mutations in the EYS gene. Doc Ophthalmol 2014;128:211-217.

42. Ratnapriya R, Swaroop A. Genetic architecture of retinal and macular degenerative diseases: the promise and challenges of next-generation sequencing. Genome Med 2013;11:1-14.

43. Katagiri S, Hayashi T, Yoshitake K, et al. Novel C8orf37 mutations in patients with early-onset retinal dystrophy, macular atrophy, cataracts, and high myopia. Ophthalmic Genet 2014;12:1-8.

44. Estrada-Cuzcano A, Neveling K, Kohl S, et al.; European Retinal Disease Consortium. Mutations in C8orf37, encoding a ciliary protein, are associated with autosomal-recessive retinal dystrophies with early macular involvement. Am J Hum Genet 2012;90:102-109.

45. van Huet RA, Estrada-Cuzcano A, Banin E, et al. Clinical characteristics of rod and cone photoreceptor dystrophies in patients with mutations in the C8orf37 gene. Invest Ophthalmol Vis Sci 2013;54:4683-4690.

46. Goldenberg-Cohen N, Banin E, Zalzstein Y, et al. Genetic heterogeneity and consanguinity lead to a "double hit": homozygous mutations of MYO7A and PDE6B in a patient with retinitis pigmentosa. Mol Vis 2013;19: 1565-1571

47. Prokudin I, Li D, He S, et al. Value of whole exome sequencing for syndromic retinal dystrophy diagnosis in young patients. Clin Experiment Ophthalmo/; e-pub ahead of print 24 July 2014

48. Landrum MJ, Lee JM, Riley GR, et al. ClinVar: public archive of relationships among sequence variation and human phenotype. Nucleic Acids Res 2014;42(Database issue):D980-D985.

49. Bamshad MJ, Ng SB, Bigham AW, et al. Exome sequencing as a tool for Mendelian disease gene discovery. Nat Rev Genet 2011;12:745-755.

50. Sherry ST, Ward MH, Kholodov M, et al. dbSNP: the NCBI database of genetic variation. Nucleic Acids Res 2001;29:308-311.

51. Exome Variant Server, NHLBI GO Exome Sequencing Project (ESP), Seattle, WA http://evs.gs.washington.edu/EVS/. Accessed 18 November 2014.

52. Exome Aggregation Consortium (ExAC), Cambridge, MA. http://exac. broadinstitute.org. Accessed 18 November 2014.

53. Aziz N, Zhao Q, Bry L, et al. College of American Pathologists' Laboratory Standards for Next-Generation Sequencing Clinical Tests. Arch Pathol Lab Med; e-pub ahead of print 25 August 2014.

54. Atwal PS, Brennan ML, Cox R, et al. Clinical whole-exome sequencing: are we there yet? Genet Med 2014;16:717-719.

55. Rehm HL, Bale SJ, Bayrak-Toydemir P, et al.; Working Group of the American College of Medical Genetics and Genomics Laboratory Quality Assurance Commitee. ACMG clinical laboratory standards for next-generation sequencing. Genet Med 2013;15:733-747.

56. Bell CJ, Dinwiddie DL, Miller NA, et al. Carrier testing for severe childhood recessive diseases by next-generation sequencing. Sci Trans/ Med 2011;3:65ra4.

57. Berg JS, Adams M, Nassar N, et al. An informatics approach to analyzing the incidentalome. Genet Med 2013;15:36-44.

58. Dorschner MO, Amendola LM, Turner EH, et al.; National Heart, Lung, and Blood Institute Grand Opportunity Exome Sequencing Project. Actionable, pathogenic incidental findings in 1,000 participants' exomes. Am J Hum Genet 2013;93:631-640.

59. Webster AR, Héon E, Lotery AJ, et al. An analysis of allelic variation in the ABCA4 gene. Invest Ophthalmo/ Vis Sci 2001;42:1179-1189.

60. Biesecker LG, Green RC. Diagnostic clinical genome and exome sequencing. $N$ Engl J Med 2014;370:2418-2425.

61. Schrijver I, Aziz N, Farkas DH, et al. Opportunities and challenges associated with clinical diagnostic genome sequencing: a report of the Association for Molecular Pathology. J Mol Diagn 2012;14:525-540.

62. Bassuk AG, Sujirakul T, Tsang SH, Mahajan VB. A novel RPGR mutation masquerading as Stargardt disease. Br J Ophthalmo/ 2014;98:709-711.

63. Berg JS, Khoury MJ, Evans JP. Deploying whole genome sequencing in clinical practice and public health: meeting the challenge one bin at a time. Genet Med 2011:13:499-504.

64. Green RC, Berg JS, Grody WW, et al.; American College of Medical Genetics and Genomics. ACMG recommendations for reporting of incidental findings in clinical exome and genome sequencing. Genet Med 2013; 15:565-574.

65. Bamshad MJ, Shendure JA, Valle D, et al.; Centers for Mendelian Genomics. The Centers for Mendelian Genomics: a new large-scale initiative to identify the genes underlying rare Mendelian conditions. Am J Med Genet A 2012:158A:1523-1525 